\title{
Contribution of Nontraditional Lipid Profiles to Hyperuricemia in Rural Chinese Hypertensive Population: Findings From the China Hypertension Registry Study
}

Yu Yu

Nanchang University Second Affiliated Hospital https://orcid.org/0000-0001-7355-2437

Tian Lan

Nanchang University Second Affiliated Hospital

Dandan Wang

Nanchang University Second Affiliated Hospital

Wangsheng Fang

wuyuan county health committee

\section{Yu Tao}

Nanchang University Second Affiliated Hospital

Minghui Li

Nanchang University Second Affiliated Hospital

Xiao Huang

Nanchang University Second Affiliated Hospital

Wei Zhou

Nanchang University Second Affiliated Hospital

\section{Tao Wang}

nanchang university second affiliated hospital

\section{Lingjuan Zhu}

Nanchang University Second Affiliated Hospital

Huihui Bao

Nanchang University Second Affiliated Hospital

Xiaoshu Cheng ( $\nabla$ xiaoshumenfan126@163.com )

Department of Cardiovascular Medicine, the Second Affiliated Hospital of Nanchang University, Nanchang of Jiangxi, China. No. 1 Minde Road, Nanchang of Jiangxi, 330006, China.

Research 
Keywords: Nontraditional lipid profiles, Hyperuricemia, Hypertension, Lipid ratios, High-density lipoprotein cholesterol, Rural China

Posted Date: November 5th, 2020

DOI: https://doi.org/10.21203/rs.3.rs-101773/v1

License: (1) This work is licensed under a Creative Commons Attribution 4.0 International License. Read Full License 


\section{Abstract}

Background: Current studies support nontraditional lipid profiles [total cholesterol (TC)/high-density lipoprotein cholesterol (HDL-C) ratio, triglyceride (TG)/HDL-C ratio, low-density lipoprotein cholesterol (LDL -C)/HDL-C ratio, non-HDL-C] as reliable indicators of cardiovascular disease, stroke and diabetes. However, whether nontraditional lipid profiles can be used as reliable markers for hyperuricemia (HUA) remains unclear due to limited research. The present study investigated the relationship of nontraditional lipid profiles with HUA in hypertensive patients.

Methods: We analyzed data from 13,721 Chinese hypertensive population untreated with lipid-lowering drugs. The relationship between non-traditional lipid profiles and HUA was examined by multivariate logistic regression analysis and smooth curve fitting (penalized spline method).

Results: The results showed that there were positive associations of TC/HDL-C ratio, TG/HDL-C ratio, LDL-C/HDL-C ratio, and non-HDL-C with HUA, respectively (all $P<0.001$ ). Furthermore, nontraditional lipid profiles were converted from continuous variables to tertiles. Compared with lowest tertile, the multivariate adjusted ORs $(95 \% \mathrm{Cl})$ of TC/HDL-C ratio, TG/HDL-C ratio, LDL-C/HDL-C ratio and non-HDL-C in highest tertile were $1.79(1.62,1.99), 2.09(1.88,2.32), 1.67(1.51,1.86), 1.93(1.74,2.13)$, respectively (all $P<0.001$ ).

Conclusions: In Chinese hypertensive population, there were positive associations between nontraditional lipid profiles (TC/HDL-C ratio, TG/HDL-C ratio, LDL-C/HDL-C ratio, and non-HDL-C) and HUA. Our findings further expand the scope of application of nontraditional lipid profiles. These novel and important results suggest that nontraditional lipid profiles can be used as potential and valuable indicators of HUA, and provide a new strategy for the prevention and treatment of HUA.

\section{Background}

Hyperuricemia (HUA) is a metabolic abnormality syndrome caused by disorders of purine metabolism[1]. Previous studies have shown that HUA is an independent risk factor for metabolic syndrome, chronic kidney disease (CKD), hypertension, cardiovascular disease (CVD), type 2 diabetes mellitus (T2DM), and death[2, 3]. Epidemiological studies show that the overall prevalence of HUA in China was $13.3 \%[4]$, and the prevalence of HUA was significantly higher in hypertensive patients[5]. The dramatically increasing prevalence of HUA is a great challenge to public health concerns and constitutes a serious socioeconomic burden[6]. Hence, identifying HUA-related risk factors in hypertension population and finding potential valuable indicators, could help to improve the management and treatment strategy of chronic diseases.

Previous studies have reported that HUA was associated with higher levels of TC, TG and LDL-C, and lower levels of HDL-C[7, 8]. However, the fact is that most HUA patients usually have multiple disorders of lipid metabolism[9, 10], so a single lipid may not be an effective indicator of HUA. Recently, there is increasing evidences that nontraditional lipid profiles, represented TC/HDL-C ratio, TG/HDL-C ratio, LDL- 
C/HDL-C ratio, and non-HDL-C, are valuable and excellent markers of CVD, T2DM, CKD and all-cause mortality[11-14]. HUA, as an independent risk factor for CKD, CVD and all-cause mortality, plays an important role in the occurrence and progression of these diseases $[15,16]$. Therefore, there may be relationships between nontraditional lipid profiles and HUA. However, to date, there is limited information on the association between these four nontraditional lipid profiles and HUA in Chinese population. In addition, more than $1 / 3$ of hypertensive patients have HUA, and these two diseases have a synergistic effect on the occurrence and development of CVD and death $[17,18]$. Therefore, the present study aims to investigate the independent relationships between four nontraditional lipids profiles and HUA in rural Chinese hypertensive population.

\section{Methods}

\section{Study design and participants}

The data we analyzed came from the Chinese Hypertension Registry Study (http://www.chictr.org.cn/, No: ChiCTR1800017274), and details about the purpose, protocol, and outcome of the study have been described[19]. The inclusion and exclusion criteria for the study are described in detail in Table S1. In short, this study is a large observational cohort study of patients with hypertension. The aim of the study was to explore the prevalence of hypertension in China and the risk factors that influence its treatment and prognosis. Hypertension was defined as Systolic blood pressure (SBP) values $\geq 140 \mathrm{mmHg}$ and/or diastolic BP (DBP) values $\geq 90 \mathrm{mmHg}$, self-report history of hypertension, or the use of antihypertensive drug(s) at baseline[20]. From March to August 2018, a total of 14,268 patients with hypertension were recruited into our study in Wuyuan, Jiangxi Province, China, and all study participants signed informed consent before being recruited into the study. In our study, we excluded participants who were not treated with lipid-lowering drugs and finally analyzed data from 13,721 hypertensive patients. The study was conducted in accordance with the Declaration of Helsinki and was approved by the Ethics Committee of the Anhui Medical University Biomedical Institute (No. CH1059).

\section{Data collection}

In the current study, staffs with physician qualifications collected venous fasting blood for each study participant. The collected blood samples were frozen and transported to the Biaojia Biotechnology Laboratory in Shenzhen, China. Laboratory staff tested the biochemical indicators in the blood samples by an automatic clinical analyzer (Beckman Coulter, USA). Laboratory indicators include lipids, liver function, renal function, Hcy, blood glucose. Details of biochemical indicators were shown in Table 1. Body mass index (BMI) was calculated by dividing weight by the square of height $(\mathrm{kg} / \mathrm{m} 2)$. After a 10minute rest, each participant's blood pressure and heart rate were measured by an electronic sphygmomanometer. Other covariates were obtained through questionnaires, including age, sex, smoking and drinking status, disease and medication history. The specific contents of the questionnaire were presented in Table 1.

\section{Definition of nontraditional lipid profiles and HUA}


The nontraditional lipid profiles represented TC/HDL-C ratio, TG/HDL-C ratio, LDL-C/HDL-C ratio and nonHDL-C[13, 14, 21, 22]. The TC/HDL-C, TG/HDL-C and LDL-C/HDL-C ratios were calculated as TC, TG, LDL$C$ divided by HDL-C, respectively. Non-HDL-C was calculated as HDL-C subtracted from TC. To date, eight guideline documents recommended that serum uric acid level $>420 \mu \mathrm{mol} / \mathrm{L}(7 \mathrm{mg} / \mathrm{dL})$ was diagnosed as HUA, regardless of sex[23-25]. According to the updated Chinese guidelines, HUA in our study was defined as serum uric acid level $>420 \mu \mathrm{mol} / \mathrm{L}(7 \mathrm{mg} / \mathrm{dL})$, regardless of gender[25].

\section{Statistical analysis}

Baseline characteristics of the study population were displayed according to with or without HUA. Continuous variables are presented as the mean \pm SD and categorical variables are presented as percentage (\%). Logistic regression analyses models were performed to assess the association of nontraditional lipid profiles (TC/HDL-C ratio, TG/HDL-C ratio, LDL-C/HDL-C ratio, and non-HDL-C) with HUA by presenting the odds ratio (OR) and $95 \%$ confidence interval $(\mathrm{Cl})$ after adjusting for confounding factors. The trend test was used to evaluate the linear relationship between nontraditional lipid profiles and HUA, when nontraditional lipid profiles used as tertiles. Fully adjusted smoothing curve fitting (penalized spline method) visually demonstrated the relationship between nontraditional lipid profiles and HUA.

All statistical analyses were performed using the statistical package R (http://www.R-project.org, The R Foundation) and Empower (R) (http://www.empowerstats.com, X\&Y Solutions, Inc., Boston, MA). Statistical significance was defined as two-tailed $P<0.05$.

\section{Results}

\section{Baseline characteristics of the study participants}

A total of 13,721 hypertensive patients untreated lipid-lowering drugs were included in our analysis (mean age: $63.79 \pm 9.41$ years; $47.23 \%$ males) (Fig. 1). The distributions of study participant baseline characteristics according to the status of HUA (non-HUA and HUA) were presented in Table 1. Compare with non-HUA group, HUA group had higher values for age, male, current smoking, alcohol use, CHD, diabetes mellitus, antihypertensive drugs, BMI, DBP, TC, TG, LDL-C, TC/HDL-C ratio, TG/HDL-C ratio, LDL$\mathrm{C} / \mathrm{HDL}-\mathrm{C}$ ratio, non-HDL-C, Hcy and FBG, and lower values for SBP, HDL-C and eGFR (all $\mathrm{P}<0.05)$.

\section{Relationship between nontraditional lipid profiles and HUA}

Fig. 2 showed the results of smooth curves between four nontraditional lipids profiles and HUA. Fig. 2A, $2 \mathrm{~B}, 2 \mathrm{C}$ and $2 \mathrm{D}$ showed the positive associations of TC/HDL-C ratio, TG/HDL-C ratio, LDL-C/HDL-C ratio and non-HDL-C with HUA, respectively.

Table 2 showed the results of multiple logistic regression analysis of the relationship between nontraditional lipid profiles and HUA. In the crude and adjusted model, there were positive associations of TC/HDL-C ratio, TG/HDL-C ratio, LDL-C/HDL-C ratio, and non-HDL-C with HUA, respectively (all $P<0.001$ ). 
After adjusting for all variables, an increment of $1 \mathrm{SD}$ in TC/HDL-C ratio, TG/HDL-C ratio, LDL-C/HDL-C ratio, and non-HDL-C was associated with greater ORs $(95 \% \mathrm{Cl})$ of $1.36(1.29,1.43), 1.28(1.23,1.33), 1.42$ $(1.33,1.51)$, and $1.35(1.30,1.41)$ for HUA, respectively (all $P<0.001)$. Furthermore, nontraditional lipid profiles were converted from continuous variables to tertiles. Compared with group $\mathrm{T} 1$, the multivariate adjusted ORs $(95 \% \mathrm{Cl})$ of TC/HDL-C ratio, TG/HDL-C ratio, LDL-C/HDL-C ratio and non-HDL-C in group T3 were 1.79 (1.62, 1.99), 2.09 (1.88, 2.32), $1.67(1.51,1.86), 1.93(1.74,2.13)$, respectively (all $P<0.001)$. All $P$ for trend $<0.001$ indicated the associations between four nontraditional lipid profiles and HUA were likely to be linear.

\section{Discussion}

In this large sample of Chinese hypertensive population, the constellation of our findings offered novel evidence for an independent positive association of nontraditional lipid profiles (TC/HDL-C ratio, TG/HDL-C ratio, LDL-C/HDL-C ratio, and non-HDL-C) with HUA. Our findings suggest that detection of nontraditional lipid profiles may be beneficial for the prevention and treatment of HUA.

Increasing evidence suggested that nontraditional lipid profiles could be a valuable indicator for a variety of diseases. Guo et al. [13] included 5,782 patients with hypertension (mean age: $57 \pm 10$ years) for analysis, and found that higher TC/HDL-C, TG/HDL-C and LDL-C/HDL-C ratio were associated with higher risk of ischemic stroke, while non-HDL-C was not associated with ischemic stroke. Wang et al. [22] analyzed data from 3,259 patients with hypertension (mean age: $58.78 \pm 10.20$ years), and found that nontraditional lipid profiles were positively correlated with reduced eGFR. Wang et al. [21] analyzed data of 10,756 Chinese (mean age: 53.8 years) and found that nontraditional lipid profiles were positively correlated with CVD risk. Wang et al.[14] analyzed the data of 2,944 hypertensive patients (mean age: $57.09 \pm 11.29$ years), indicating that the nontraditional lipid profiles were significantly positively associated with diabetes, and further found that TG/HDL-C ratio was more significantly correlated with diabetes. Therefore, we have sufficient reasons to believe that nontraditional lipid profiles are effective indicators of these diseases, including stroke, CVD, diabetes, CKD. It is noteworthy that HUA plays an important role in the occurrence and development of the diseases mentioned above[3,24,26]. Therefore, prevention and treatment of HUA have great clinical benefits. However, to our knowledge, few studies have explored the relationship between nontraditional lipid profiles and HUA, so it is not clear whether nontraditional lipid profiles can serve as valuable markers of HUA. Our findings extend the application of nontraditional lipids and well fill in the gaps of previous studies, so this study has very important clinical significance.

There is a panel of plausible pathomechanisms responsible for the increased risk of HUA in those with poor nontraditional lipid profiles. It was widely-accepted that reduced renal function, inflammation, insulin resistance (IR), lifestyle and lipid-lowering drugs can induce HUA. Excessive lipids deposit on the intima, and mononuclear macrophages become foam cells by phagocytosis of lipids deposited on the intima, thus causing renal arteriosclerosis, resulting in reduced filtration function of the kidney, and thus reduced excretion of uric acid from the kidney, resulting in HUA production[27]. Higher levels of TC/HDL-C and 
TG/HDL-C ratio may be mediated by CRP-mediated inflammatory response, leading to the occurrence of HUA $[28,29]$. Higher lipids lead to IR through lipotoxicity, inflammation and endoplasmic reticulum stress, which in turn leads to the development of HUA. In addition, some researchers have found that hyperlipidemia and HUA patients share the same lifestyle and diet, such as excessive alcohol consumption and high-fat food intake, which also suggests that clinicians should conduct lipid-lowering and uric acid-lowering treatments simultaneously[30]. Interestingly, Deedwania et al. found that lipidlowering drugs not only reduced lipid levels, but also reduced SUA in patients with CVD[31]. There was a close relationship between nontraditional lipid profiles and HUA. However, current basic studies cannot fully elucidate the mechanism behind this relationship, and further basic experiments are needed to fully elucidate the specific biological mechanism of this association.

\section{Study Strengths And Limitations}

Our study has obvious strengths and some limitations. This study is currently the first study to examine the association of nontraditional lipid profiles (TC/HDL-C ratio, TG/HDL-C ratio, LDL-C/HDL-C ratio, and non-HDL-C) with the risk of HUA in Chinese hypertension population. Nevertheless, some limitations should be noted. First, as a cross-sectional study, our results failed to provide causality regarding the relationship between nontraditional lipid profiles and HUA. Second, our study population is from Chinese hypertensive patients, so the generality of our conclusions is limited.

\section{Conclusions}

In rural Chinese hypertensive population, there were positive associations between nontraditional lipid profiles (TC/HDL-C ratio, TG/HDL-C ratio, LDL-C/HDL-C ratio, and non-HDL-C) and HUA. Our findings further expand the scope of application of nontraditional lipid profiles. These novel and important results suggest that nontraditional lipid profiles can be used as potential and valuable indicators of HUA, and provide a new strategy for the prevention and treatment of HUA.

\section{Abbreviations}

CKD: Chronic kidney disease; CVD: Cardiovascular disease; T2DM: Type 2 diabetes mellitus; CHD: Coronary heart disease; BMI: Body mass index; SBP: Systolic blood pressure; DBP: Diastolic blood pressure; TC: Total cholesterol; TG: Triglyceride; LDL-C: Low density lipoprotein cholesterol; HDL-C: High density lipoprotein cholesterol; Hcy: Homocysteine; FBG: Fasting blood glucose; Egfr: estimated glomerular filtration rate.

\section{Declarations}

Acknowledgements 
All authors acknowledge the contribution the all staff who participated in this study as well as the study participants who shared their time with us.

\section{Author contributions}

All authors were responsible for drafting the manuscript and revising it critically for constructive intellectual content. All authors approved the version to be published.

\section{Funding}

This study was supported by Science and Technology Innovation Platform Project of Jiangxi Province (Grant number: 20165BCD41005), and the Science and Technology Plan of Health Commission of Jiangxi Province (Grant number: 20185215).

\section{Availability of data and materials}

The datasets used and/or analyzed during the current study are available from the corresponding author on reasonable request.

\section{Ethics approval and consent to participate}

The study was conducted in accordance with the Declaration of Helsinki and was approved by the Ethics Committee of the Anhui Medical University Biomedical Institute (No. CH1059). Informed written consent was obtained from all patients before their enrollment in this study.

\section{Consent for publication}

Not applicable.

\section{Competing interests}

The authors declare that they have no conflict of interest.

\section{References}

1. Dalbeth N, Merriman TR, Stamp LK. Gout. Lancet. 2016;10055:2039-2052.

2. Bardin T, Richette P. Impact of comorbidities on gout and hyperuricaemia: an update on prevalence and treatment options. Bmc Med. 2017;1:123.

3. Zhu Y, Pandya BJ, Choi HK. Comorbidities of gout and hyperuricemia in the US general population: NHANES 2007-2008. Am J Med. 2012;7:679-687.

4. Liu R, Han C, Wu D, Xia X, Gu J, Guan H, Shan Z, Teng W. Prevalence of Hyperuricemia and Gout in Mainland China from 2000 to 2014: A Systematic Review and Meta-Analysis. Biomed Res Int. 2015:762820. 
5. Krishnan E, Kwoh CK, Schumacher HR, Kuller L. Hyperuricemia and incidence of hypertension among men without metabolic syndrome. Hypertension. 2007;2:298-303.

6. Xia Y, Wu Q, Wang H, Zhang S, Jiang Y, Gong T, Xu X, Chang Q, Niu K, Zhao Y. Global, regional and national burden of gout, 1990-2017: a systematic analysis of the Global Burden of Disease Study. Rheumatology (Oxford). 2020;7:1529-1538.

7. Liang J, Jiang Y, Huang Y, Song W, Li X, Huang Y, Ou J, Wei Q, Gu J. The comparison of dyslipidemia and serum uric acid in patients with gout and asymptomatic hyperuricemia: a cross-sectional study. Lipids Health Dis. 2020;1:31.

8. Yu Y, Huang X, Li M, Ding C, Hu L, Zhong X, Zhou W, Wang T, Zhu L, Bao H et al. Is There a Nonlinear Relationship between Serum Uric Acid and Lipids in a Hypertensive Population with eGFR $>/=30$ $\mathrm{ml} / \mathrm{min} / 1.73 \mathrm{~m}(2)$ ? Findings from the China Hypertension Registry Study. Int J Endocrinol. 2020:9725979.

9. Li Q, Yang Z, Lu B, Wen J, Ye Z, Chen L, He M, Tao X, Zhang W, Huang Y et al. Serum uric acid level and its association with metabolic syndrome and carotid atherosclerosis in patients with type 2 diabetes. Cardiovasc Diabetol. 2011:72.

10. Kim ES, Kwon HS, Ahn CW, Lim DJ, Shin JA, Lee SH, Cho JH, Yoon KH, Kang MI, Cha BY et al. Serum uric acid level is associated with metabolic syndrome and microalbuminuria in Korean patients with type 2 diabetes mellitus. J Diabetes Complications. 2011;5:309-313.

11. Arsenault BJ, Rana JS, Stroes ES, Despres JP, Shah PK, Kastelein JJ, Wareham NJ, Boekholdt SM, Khaw KT. Beyond low-density lipoprotein cholesterol: respective contributions of non-high-density lipoprotein cholesterol levels, triglycerides, and the total cholesterol/high-density lipoprotein cholesterol ratio to coronary heart disease risk in apparently healthy men and women. J Am Coll Cardiol. 2009;1:35-41.

12. Bittner V, Johnson BD, Zineh I, Rogers WJ, Vido D, Marroquin OC, Bairey-Merz CN, Sopko G. The triglyceride/high-density lipoprotein cholesterol ratio predicts all-cause mortality in women with suspected myocardial ischemia: a report from the Women's Ischemia Syndrome Evaluation (WISE). Am Heart J. 2009;3:548-555.

13. Guo X, Li Z, Sun G, Guo L, Zheng L, Yu S, Yang H, Pan G, Zhang Y, Sun Y. Comparison of four nontraditional lipid profiles in relation to ischemic stroke among hypertensive Chinese population. Int J Cardiol. 2015:123-125.

14. Wang H, Guo X, Chen Y, Li Z, Xu J, Sun Y. Relation of four nontraditional lipid profiles to diabetes in rural Chinese H-type hypertension population. Lipids Health Dis. 2017;1:199.

15. loannou GN, Boyko EJ. Effects of menopause and hormone replacement therapy on the associations of hyperuricemia with mortality. Atherosclerosis. 2013;1:220-227.

16. Chen JH, Chuang SY, Chen HJ, Yeh WT, Pan WH. Serum uric acid level as an independent risk factor for all-cause, cardiovascular, and ischemic stroke mortality: a Chinese cohort study. Arthritis Rheum. 2009;2:225-232.

17. Trachtman H. Treatment of hyperuricemia in essential hypertension. Hypertension. 2007;6:e45, e46. 
18. Yu Y, Li M, Zhou W, Wang T, Zhu L, Hu L, Bao H, Cheng X. Diastolic blood pressure achieved at target systolic blood pressure $(120-140 \mathrm{~mm} \mathrm{Hg})$ and dabigatran-related bleeding in patients with nonvalvular atrial fibrillation: A real-world study. Anatol J Cardiol. 2020;4:267-273.

19. Yu Y, Hu L, Huang X, Zhou W, Bao H, Cheng X. BMI modifies the association between serum HDL cholesterol and stroke in a hypertensive population without atrial fibrillation. J Endocrinol Invest. 2020.

20. 2018 Chinese Guidelines for Prevention and Treatment of Hypertension-A report of the Revision Committee of Chinese Guidelines for Prevention and Treatment of Hypertension. J Geriatr Cardiol. 2019;3:182-241.

21. Wang H, Li Z, Guo X, Chen Y, Chang Y, Chen S, Sun Y. The impact of nontraditional lipid profiles on left ventricular geometric abnormalities in general Chinese population. BMC Cardiovasc Disord. 2018;1:88.

22. Wang H, Li Z, Guo X, Chen Y, Chen S, Tian Y, Sun Y. Contribution of non-traditional lipid profiles to reduced glomerular filtration rate in $\mathrm{H}$-type hypertension population of rural China. Ann Med. 2018;3:249-259.

23. Li Q, Li X, Wang J, Liu H, Kwong JS, Chen H, Li L, Chung SC, Shah A, Chen Y et al. Diagnosis and treatment for hyperuricemia and gout: a systematic review of clinical practice guidelines and consensus statements. Bmj Open. 2019;8:e26677.

24. FitzGerald JD, Dalbeth N, Mikuls T, Brignardello-Petersen R, Guyatt G, Abeles AM, Gelber AC, Harrold LR, Khanna D, King C et al. 2020 American College of Rheumatology Guideline for the Management of Gout. Arthritis Rheumatol. 2020;6:879-895.

25. Chinese Multidisciplinary Expert Consensus on the Diagnosis and Treatment of Hyperuricemia and Related Diseases. Chin Med J (Engl). 2017;20:2473-2488.

26. Kanbay M, Segal M, Afsar B, Kang DH, Rodriguez-Iturbe B, Johnson RJ. The role of uric acid in the pathogenesis of human cardiovascular disease. Heart. 2013;11:759-766.

27. Vaziri ND, Norris K. Lipid disorders and their relevance to outcomes in chronic kidney disease. Blood Purif. 2011;1-3:189-196.

28. Agirbasli M, Tanrikulu A, Acar SB, Azizy M, Bekiroglu N. Total cholesterol-to-high-density lipoprotein cholesterol ratio predicts high-sensitivity C-reactive protein levels in Turkish children. $\mathrm{J}$ Clin Lipidol. 2015;2:195-200.

29. Cushman M, Arnold AM, Psaty BM, Manolio TA, Kuller LH, Burke GL, Polak JF, Tracy RP. C-reactive protein and the 10-year incidence of coronary heart disease in older men and women: the cardiovascular health study. Circulation. 2005;1:25-31.

30. Conen D, Wietlisbach V, Bovet P, Shamlaye C, Riesen W, Paccaud F, Burnier M. Prevalence of hyperuricemia and relation of serum uric acid with cardiovascular risk factors in a developing country. Bmc Public Health. 2004:9.

31. Deedwania PC, Stone PH, Fayyad RS, Laskey RE, Wilson DJ. Improvement in Renal Function and Reduction in Serum Uric Acid with Intensive Statin Therapy in Older Patients: A Post Hoc Analysis of 
the SAGE Trial. Drugs Aging. 2015;12:1055-1065.

\section{Tables}




\section{Table 1. Characteristics of Study Population}

Variables* Total $(n=13,721) \quad$ non-HUA $(n=7,622) \quad$ HUA $(n=6,099) \quad P$ value

\section{Demographics}

\begin{tabular}{|c|c|c|c|c|}
\hline Age, years & $63.79 \pm 9.41$ & $63.56 \pm 9.02$ & $64.08 \pm 9.87$ & 0.002 \\
\hline Male, & $6,484(47.23)$ & 2,525 (33.13) & $3,954(64.83)$ & $<0.001$ \\
\hline Current smoking, $\%$ & $3,568(26.00)$ & $1,578(20.71)$ & $1,987(32.58)$ & $<0.001$ \\
\hline Alcohol use, \% & $3,010(21.93)$ & $1,206(15.83)$ & $1,801(29.53)$ & $<0.001$ \\
\hline \multicolumn{5}{|l|}{ Comorbidity, \% } \\
\hline Stroke & $820(5.97)$ & $450(5.90)$ & $370(6.07)$ & 0.69 \\
\hline $\mathrm{CHD}$ & $633(4.61)$ & $321(4.21)$ & $312(5.12)$ & 0.012 \\
\hline Diabetes mellitus & $2,438(17.76)$ & $1,250(16.40)$ & $1,187(19.46)$ & $<0.001$ \\
\hline \multicolumn{5}{|l|}{ Medication use, \% } \\
\hline Antihypertensive drugs & $8,788(64.03)$ & $4,725(62.01)$ & $4,059(66.56)$ & $<0.001$ \\
\hline Glucose-lowering drugs & $662(4.82)$ & $361(4.74)$ & $300(4.92)$ & 0.62 \\
\hline \multicolumn{5}{|l|}{ Physical examination } \\
\hline $\mathrm{BMI}, \mathrm{kg} / \mathrm{m}^{2}$ & $23.57 \pm 3.75$ & $23.25 \pm 3.82$ & $23.98 \pm 3.61$ & $<0.001$ \\
\hline SBP, mmHg & $148.54 \pm 17.82$ & $149.45 \pm 17.31$ & $147.39 \pm 18.39$ & $<0.001$ \\
\hline DBP, $\mathrm{mmHg}$ & $89.04 \pm 10.76$ & $88.75 \pm 10.39$ & $89.40 \pm 11.20$ & $<0.001$ \\
\hline \multicolumn{5}{|l|}{ Laboratory results } \\
\hline $\mathrm{TC}, \mathrm{mmol} / \mathrm{L}$ & $5.18 \pm 1.10$ & $5.13 \pm 1.06$ & $5.24 \pm 1.15$ & $<0.001$ \\
\hline $\mathrm{TG}, \mathrm{mmol} / \mathrm{L}$ & $1.80 \pm 1.25$ & $1.64 \pm 1.04$ & $1.99 \pm 1.44$ & $<0.001$ \\
\hline LDL-C, mmol/L & $3.00 \pm 0.80$ & $2.96 \pm 0.78$ & $3.05 \pm 0.83$ & $<0.001$ \\
\hline $\mathrm{HDL}-\mathrm{C}, \mathrm{mmol} / \mathrm{L}$ & $1.57 \pm 0.43$ & $1.60 \pm 0.43$ & $1.53 \pm 0.42$ & $<0.001$ \\
\hline TC/HDL-C ratio & $3.44 \pm 0.87$ & $3.33 \pm 0.81$ & $3.58 \pm 0.92$ & $<0.001$ \\
\hline TG/HDL-C ratio & $1.31 \pm 1.27$ & $1.16 \pm 1.04$ & $1.50 \pm 1.48$ & $<0.001$ \\
\hline LDL-C/HDL-C ratio & $2.01 \pm 0.65$ & $1.94 \pm 0.62$ & $2.10 \pm 0.69$ & $<0.001$ \\
\hline non-HDL-C, mmol/L & $3.61 \pm 0.99$ & $3.53 \pm 0.93$ & $3.71 \pm 1.04$ & $<0.001$ \\
\hline $\mathrm{Hcy}, \mu \mathrm{mol} / \mathrm{L}$ & $17.96 \pm 11.03$ & $16.28 \pm 9.23$ & $20.06 \pm 12.63$ & $<0.001$ \\
\hline $\mathrm{FBG}, \mathrm{mmol} / \mathrm{L}$ & $6.17 \pm 1.59$ & $6.14 \pm 1.68$ & $6.20 \pm 1.47$ & 0.027 \\
\hline
\end{tabular}


Abbreviations: CHD, coronary heart disease; BMI, body mass index; SBP, systolic blood pressure; DBP, diastolic blood pressure; TC, total cholesterol; TG, triglyceride; LDL-C, low density lipoprotein cholesterol; HDL-C, high density lipoprotein cholesterol; Hcy, homocysteine; FBG, fasting blood glucose; eGFR, estimated glomerular filtration rate.

*Data are presented as number (\%) or mean \pm standard deviation. 


\section{Table 2. Odd ratios $(95 \% \mathrm{Cl})$ for HUA according to continuous and tertiles of nontraditional lipid}

profiles

\begin{tabular}{|c|c|c|c|c|c|c|}
\hline & & & $\begin{array}{l}\text { Crude } \\
\text { model }\end{array}$ & & $\begin{array}{l}\text { Adjusted } \\
\text { model }\end{array}$ & \\
\hline Variables & $\mathrm{N}$ & $\begin{array}{l}\text { Events, } \mathrm{n} \\
\text { (\%) }\end{array}$ & OR $(95 \% \mathrm{Cl})$ & $\begin{array}{l}P \\
\text { value }\end{array}$ & $\begin{array}{l}\mathrm{OR} \\
(95 \% \mathrm{Cl})\end{array}$ & $\begin{array}{l}P \\
\text { value }\end{array}$ \\
\hline $\begin{array}{l}\text { TC/HDL-C ratio (Per } 1 \text { SD } \\
\text { increase) }\end{array}$ & 13,721 & $\begin{array}{l}6,099 \\
(44.5 \%)\end{array}$ & $\begin{array}{l}1.39(1.33 \\
1.44)\end{array}$ & $<0.001$ & $\begin{array}{l}1.36(1.29 \\
1.43)\end{array}$ & $<0.001$ \\
\hline \multicolumn{7}{|l|}{ Tertiles of TC/HDL-C ratio } \\
\hline $\mathrm{T} 1(<2.76)$ & 4,572 & $\begin{array}{l}1,728 \\
(37.8 \%)\end{array}$ & Reference & & Reference & \\
\hline T2 (2.76-4.13) & 4,574 & $\begin{array}{l}1,954 \\
\text { (42.7\%) }\end{array}$ & $\begin{array}{l}1.22(1.13 \\
1.33)\end{array}$ & $<0.001$ & $\begin{array}{l}1.30(1.18 \\
1.44)\end{array}$ & $<0.001$ \\
\hline $\mathrm{T} 3(\geq 4.13)$ & 4,575 & $\begin{array}{l}2,417 \\
(52.8 \%)\end{array}$ & $\begin{array}{l}1.84(1.70 \\
2.00)\end{array}$ & $<0.001$ & $\begin{array}{l}1.79(1.62, \\
1.99)\end{array}$ & $<0.001$ \\
\hline$P$ for trend & & & $<0.001$ & & $<0.001$ & \\
\hline $\begin{array}{l}\text { TG/HDL-C ratio (Per } 1 \text { SD } \\
\text { increase) }\end{array}$ & 13,721 & $\begin{array}{l}6,099 \\
(44.5 \%)\end{array}$ & $\begin{array}{l}1.27(1.23 \\
1.31)\end{array}$ & $<0.001$ & $\begin{array}{l}1.28(1.23, \\
1.33)\end{array}$ & $<0.001$ \\
\hline \multicolumn{7}{|l|}{ Tertiles of TG/HDL-C ratio } \\
\hline $\mathrm{T} 1(<0.62)$ & 4,571 & $\begin{array}{l}1,708 \\
(37.4 \%)\end{array}$ & Reference & & Reference & \\
\hline T2 (0.62-2.01) & 4,576 & $\begin{array}{l}1,934 \\
(42.3 \%)\end{array}$ & $\begin{array}{l}1.23(1.13 \\
1.33)\end{array}$ & $<0.001$ & $\begin{array}{l}1.30(1.17 \\
1.43)\end{array}$ & $<0.001$ \\
\hline $\mathrm{T} 3(\geq 2.01)$ & 4,574 & $\begin{array}{l}2,457 \\
(53.7 \%)\end{array}$ & $\begin{array}{l}1.95(1.79 \\
2.11)\end{array}$ & $<0.001$ & $\begin{array}{l}2.09(1.88 \\
2.32)\end{array}$ & $<0.001$ \\
\hline$P$ for trend & & & $<0.001$ & & $<0.001$ & \\
\hline $\begin{array}{l}\text { LDL-C/HDL-C ratio (Per } 1 \\
\text { SD increase) }\end{array}$ & 13,721 & $\begin{array}{l}6,099 \\
(44.5 \%)\end{array}$ & $\begin{array}{l}1.47(1.40 \\
1.55)\end{array}$ & $<0.001$ & $\begin{array}{l}1.42(1.33 \\
1.51)\end{array}$ & $<0.001$ \\
\hline \multicolumn{7}{|l|}{ Tertiles of LDL/HDL-C ratio } \\
\hline $\mathrm{T} 1(<1.50)$ & 4,574 & $\begin{array}{l}1,742 \\
(38.1 \%)\end{array}$ & Reference & & Reference & \\
\hline T2 (1.50-2.53) & 4,572 & $\begin{array}{l}1,967 \\
(43.0 \%)\end{array}$ & $\begin{array}{l}1.23(1.13 \\
1.33)\end{array}$ & $<0.001$ & $\begin{array}{l}1.31(1.18 \\
1.44)\end{array}$ & $<0.001$ \\
\hline T3 $(\geq 2.53)$ & 4,575 & $\begin{array}{l}2,390 \\
(52.2 \%)\end{array}$ & $\begin{array}{l}1.78(1.64 \\
1.93)\end{array}$ & $<0.001$ & $\begin{array}{l}1.67(1.51 \\
1.86)\end{array}$ & $<0.001$ \\
\hline$P$ for trend & & & $<0.001$ & & $<0.001$ & \\
\hline
\end{tabular}




\begin{tabular}{|c|c|c|c|c|c|c|}
\hline $\begin{array}{l}\text { non-HDL-C (Per } 1 \text { SD } \\
\text { increase) }\end{array}$ & 13,721 & $\begin{array}{l}6,099 \\
(44.5 \%)\end{array}$ & $\begin{array}{l}1.20(1.16 \\
1.25)\end{array}$ & $<0.001$ & $\begin{array}{l}1.35(1.30 \\
1.41)\end{array}$ & $<0.001$ \\
\hline \multicolumn{7}{|l|}{ Tertiles of non-HDL-C } \\
\hline $\mathrm{T} 1(<2.84)$ & 4,572 & $\begin{array}{l}1,849 \\
(40.4 \%)\end{array}$ & Reference & & Reference & \\
\hline T2 (2.84-4.37) & 4,543 & $\begin{array}{l}1,945 \\
(42.8 \%)\end{array}$ & $\begin{array}{l}1.10(1.01, \\
1.20)\end{array}$ & 0.022 & $\begin{array}{l}1.31(1.19, \\
1.44)\end{array}$ & $<0.001$ \\
\hline T3 ( $\geq 4.37)$ & 4,606 & $\begin{array}{l}2,305 \\
(50.0 \%)\end{array}$ & $\begin{array}{l}1.48(1.36, \\
1.60)\end{array}$ & $<0.001$ & $\begin{array}{l}1.93(1.74 \\
2.13)\end{array}$ & $<0.001$ \\
\hline$P$ for trend & & & $<0.001$ & & $<0.001$ & \\
\hline
\end{tabular}

Abbreviations: OR, odd ratio; $\mathrm{Cl}$, confidence interval; $\mathrm{TC}$, total cholesterol; TG, triglyceride; $\mathrm{HDL}-\mathrm{C}$, highdensity lipoprotein cholesterol; LDL-C, low-density lipoprotein cholesterol; non-HDL-C, non-high-density lipoprotein cholesterol.

Adjusted model: adjusted for age, sex, BMI, SBP, DBP, current smoking, alcohol use, eGFR, Hcy, diabetes mellitus and anti-hypertensive drugs.

\section{Figures}


14,268 participants were enrolled

\section{4 individuals without hypertension}

14,234 hypertensive individuals

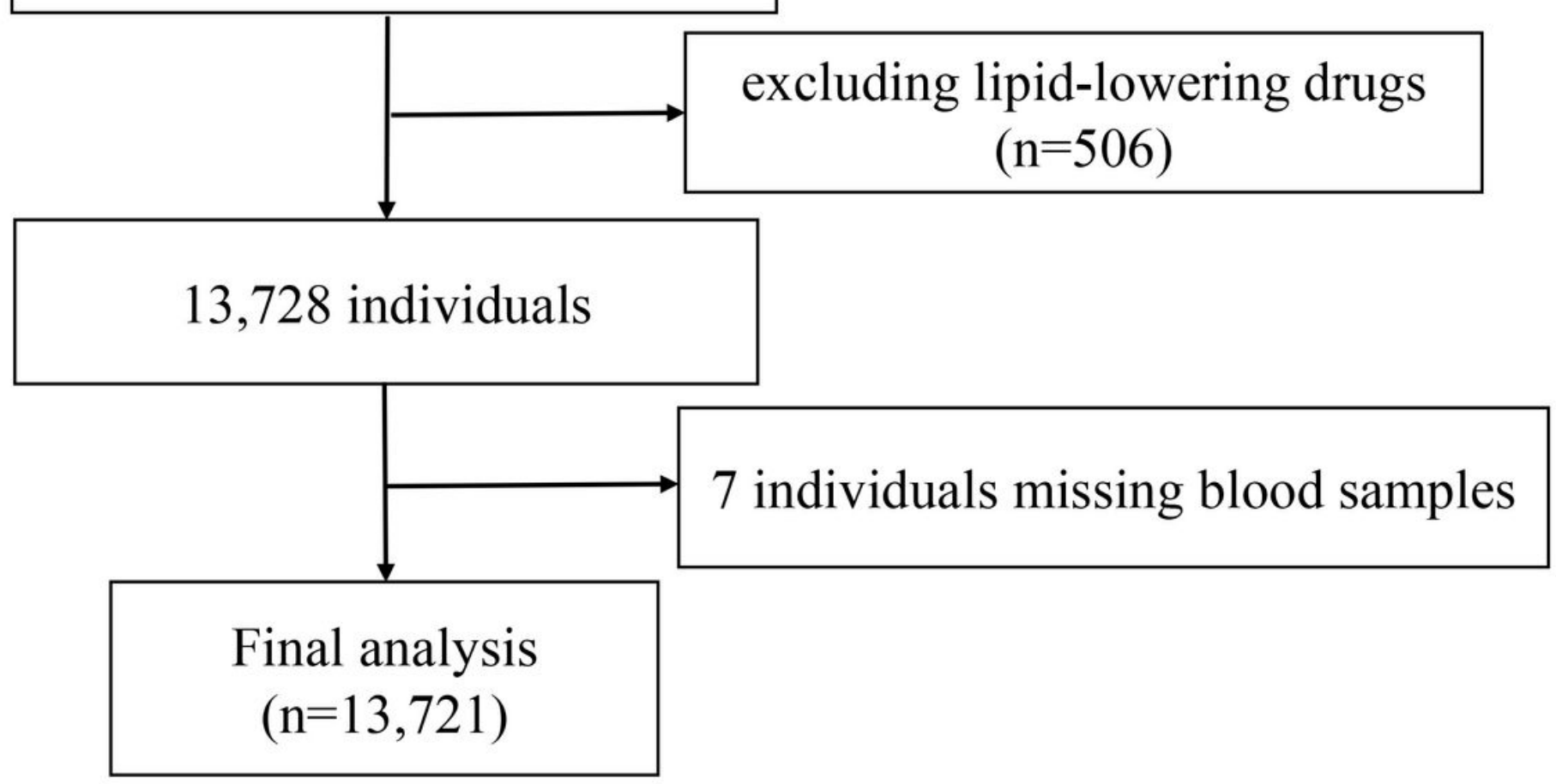

Figure 1

Flow chart of study participants 

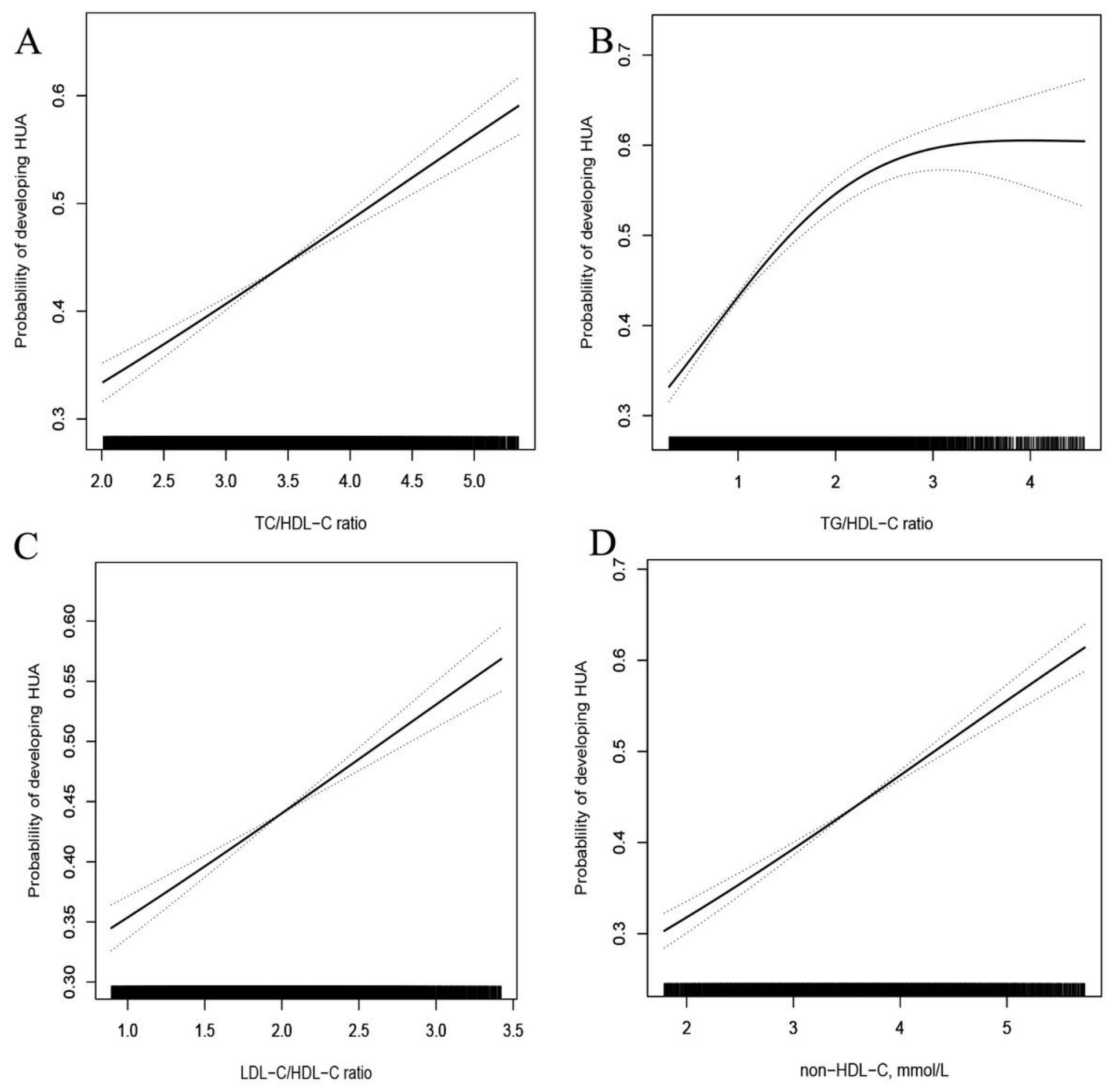

Figure 2

The association between TC/HDL-C (A), TG/HDL-C (B), LDL-C/HDL-C ratio (C), or non-HDL-C (D) and HUA. Adjusted for age, sex, BMI, SBP, DBP, current smoking, alcohol use, eGFR, Hcy, diabetes mellitus and antihypertensive drugs.

\section{Supplementary Files}


This is a list of supplementary files associated with this preprint. Click to download.

- Supplementarymaterials.docx 